\title{
Anterior Urethrectomy for Primary Carcinoma of the Female Urethra Mimicking a Urethral Caruncle
}

\author{
Ji Sung Shim, Mi Mi Oh, Jeong Gu Lee, Jae Hyun Bae \\ Department of Urology, Korea University College of Medicine, Seoul, Korea
}

\begin{abstract}
Here we report a case of primary carcinoma of the female urethra. A 52-year-old woman presented with a palpable urethral mass associated with intermittent pain that she first experienced a few months prior. Clinical examination showed a urethral mass that appeared to be a caruncle; therefore, simple carunclectomy was performed. However, on histological examination, the mass was revealed to be a squamous cell carcinoma; therefore, anterior urethrectomy was performed. During a 4-year follow-up period, the patient has been well with no dysuria, dyspareunia, or incontinence.
\end{abstract}

Keywords: Female; Urethral neoplasms; Surgery

Because of the low incidence of tumors of the female urethra, consensus on their management is lacking. Most urethral cancers are aggressive; however, partial urethrectomy can successfully control lesions that are limited to the anterior portion of the urethra. Survival is dependent on the tumor extent at the time of treatment, thus emphasizing the importance of earlier detection and careful examination of these tumors.

\section{CASE REPORT}

A 52-year-old woman with a 5-year history of a palpable urethral mass presented at Urology Department with intermittent urethral pain. The patient did not complain of any urinary symptoms or dyspareunia. Her medical history included an appendectomy 30 years previous but was otherwise unremarkable. Pelvic examination showed the presence of a urethral mass approximately $1.7 \mathrm{~cm}$ in diameter (Fig. 1). The mass appeared to be a simple urethral caruncle despite a small amount of ulceration; therefore, we did not perform urethroscopy or cystoscopy. Three hours after the carunclectomy, the Foley catheter was removed and the patient reported good voiding ability. She was then discharged, and a follow-up visit was scheduled for 7 days later.

Six days after the operation, our department received the pathological results of the excised urethral mass, which indicated a squamous cell carcinoma in situ (Fig. 2). We promptly evaluated the case for cancer and consulted with the department of gynecology. Abdominal and pelvic computed tomography, intravenous pyelography, bone scanning, and chest radiography showed no evidence of disseminated disease. The final disease stage was classified as TisNOM0; therefore, partial urethrectomy was planned.

General anesthesia was used for the surgery. A 22-Fr cystoscope was inserted through the urethra without resistance. A cystoscopic examination revealed erythematous mass-like lesions on the trigone, bladder neck (3- and 7-oclock positions), and right lateral wall. Frozen biopsy analysis showed the presence of atypical cells on the trigone and bladder neck (at two points), but no malignancy was identified on the right lateral wall. The urethral orifice was tagged with a 2-0 silk suture (12-, 3-, and 9-oclock positions), and we were able to excise the anterior urethra. The final pathologic analysis indicated a negative surgical
Corresponding author: Jae Hyun Bae

Department of Urology, Korea University Ansan Hospital, Korea University College of Medicine, 123 Jeokgeum-ro, Danwon-gu, Ansan 425-707, Korea Tel: +82-31-412-5190 / Fax: +82-31-412-5194

E-mail: urobae@genetherapy.or.kr

Submitted: October 22, 2013 / Accepted after revision: November 19, 2013
This is an Open Access article distributed under the terms of the Creative Commons Attribution Non-Commercial License (http://creativecommons.org/licenses/by-nc/3.0/) which permits unrestricted non-commercial use, distribution, and reproduction in any medium, provided the original work is properly cited. 


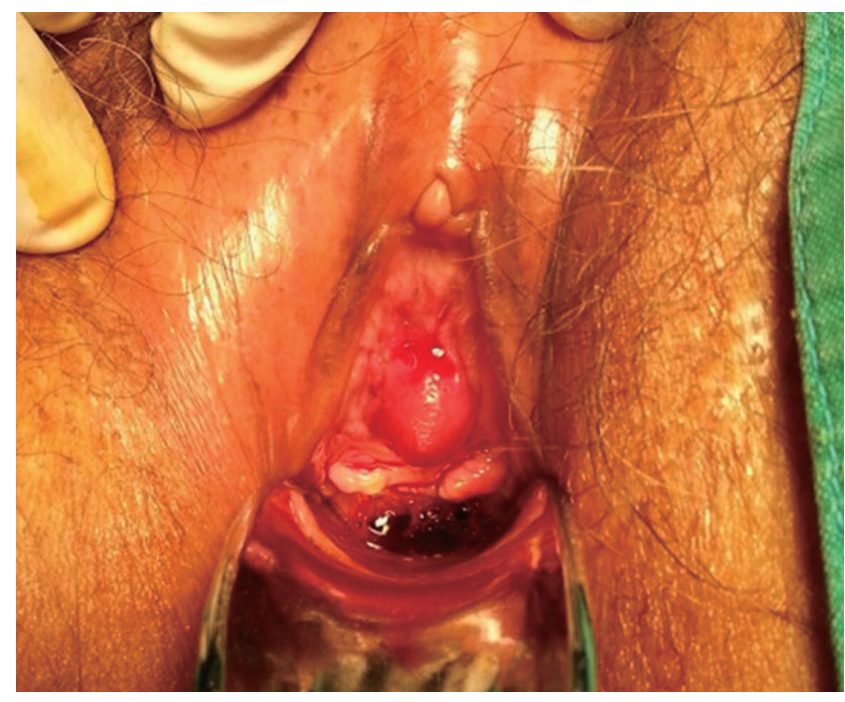

Fig. 1. A urethral mass in a postmenopausal woman.

margin and a tumor depth of $0.3 \mathrm{~cm}$.

The patient was followed up at 6-month intervals. Her medical records were assessed, and a clinical examination was performed. Imaging studies were performed when she reported new symptoms. She remained free of recurrence and reported normal voiding function 50 months after the tumor was diagnosed.

\section{DISCUSSION}

The female urethra can be anatomically divided into an anterior segment and a posterior segment. Histologically, the proximal one-third of the urethra is lined with typical transitional urothelium and the distal two-thirds is lined by stratified squamous epithelium. The histology of the carcinoma primarily depends on the site of origin within the urethra; squamous cell carcinoma is the most common histologic type, as it accounts for $50-70 \%$ of all cases. However, the treatment strategy for female urethral carcinoma is independent of the histologic type because survival rates do not appear to differ among the various histologic types [1]. In fact, the site of origin of the carcinoma and the clinical stage are important factors in determining survival.

In the case reported here, we initially excised the urethral mass becausem its features were similar to those of a urethral caruncle, with a reddish exophytic mass present at the urethral meatus that was covered with mucosa. A urethral caruncle is a benign pedunculated lesion of the urethra for which simple excision is curative. However, as many as $5 \%$ of cases of clinically

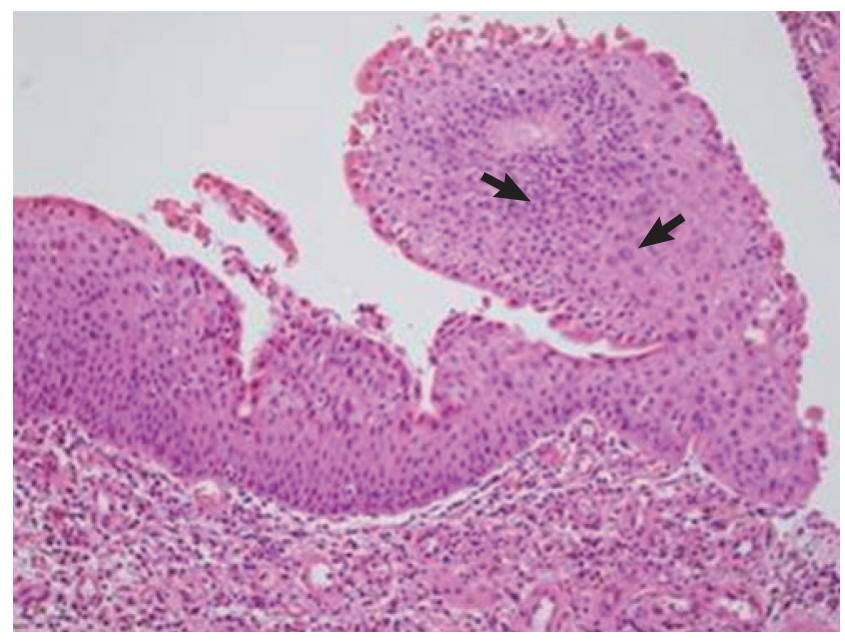

Fig. 2. Histologic features of the surgical specimen: arrows showing high-grade well-differentiated squamous cell carcinoma in situ with cells of various sizes (H\&E, ×400).

suspected caruncles may represent true neoplasms, of which approximately half are malignant [2].

Alternatively, a caruncle may subsequently develop into urethral cancer. Although the cause of urethral carcinoma in women has not yet been identified, several factors have been implicated. Etiologic factors associated with the development of urethral carcinoma include caruncle, diverticulum, polyps, parturition, and infection with the human papilloma virus or other viruses [3].

Here we performed only an anterior urethrectomy because tumors in the distal urethra tend to be low stage and high cure rates (70-90\%) can be achieved with local excision [1]. Results of the frozen biopsy revealed no malignancy on the urethral margin, confirming that excision of the bladder neck, vulva, vagina, uterus, and adnexa was nonessential.

There is currently no consensus on the treatment of women with urethral cancer. Further, these are generally slow-growing tumors with no specific symptoms or signs. They manifest late and can be misdiagnosed as urethral lesions mimicking tumors. Intestinal metaplasia, tuberculosis, and melanoma have all been reported to either coexist with or mimic urethral caruncles [4]. Therefore, in terms of appearance or behavior of atypical lesions, excision may be warranted to exclude the possibility of other entities.

Urethral carcinoma is generally aggressive, with a recurrencefree survival rate of $45 \%$ and a disease-free survival rate of $60 \%$ at 10 years after surgery [2]. Therefore, aggressive local resection via radical urethrectomy may improve local control in cases of 
low-stage cancer. However, partial urethrectomy followed by imaging studies and adequate biopsies may be sufficient in cases of slow progression or the possibility of the malignant transformation of a caruncle. Accordingly, aggressive treatment, which may be associated with a high risk of complications, can be avoided.

\section{CONFLICT OF INTEREST}

No potential conflict of interest relevant to this article was reported.

\section{REFERENCES}

1. Dimarco DS, Dimarco CS, Zincke H, Webb MJ, Bass SE, Slezak JM, et al. Surgical treatment for local control of female urethral carcinoma. Urol Oncol 2004;22:404-9.

2. Johnson DE, O'Connell JR. Primary carcinoma of female urethra. Urology 1983;21:42-5.

3. Grigsby P, Herr H. Urethral tumors. In: Vogelzang N, Shipley WU, Scardino PT, Coffey DS, editors. Comprehensive textbook of genitourinary oncology. 2nd ed. Philadelphia: Lippincott Williams \& Wilkins; 2000. p. 1133-9.

4. Indudhara R, Vaidyanathan S, Radotra BD. Urethral tuberculosis. Urol Int 1992;48:436-8. 